\title{
DIAGNÓSTICO DE ENFERMERÍA EN LA SALUD FÍSICA DE PREESCOLARES, EN UNA COMUNIDAD MEXICANA
}

\author{
Diagnosis of Nursing in the Physical Health of \\ Prestudents, in a Mexican Community \\ Diagnóstico da Enfermagem na Saúde Física \\ de Pré-escolares em uma Comunidade Mexicana
}

\section{Resumen}

Las condiciones de vida del ser humano han cambiado, con ello la salud de grupos etáreos. El individuo se expone a prácticas de salud acorde a sus patrones culturales y la influencia de las redes sociales. Este estudio identificó la salud física de preescolares en una comunidad suburbana de Toluca, México. Se entrevistaron a preescolares, contando con la autorización y presencia de los padres. La morbilidad identificada fue en infecciones respiratorias agudas, enfermedades infecciosas gastrointestinales y malnutrición con factores predisponentes de prácticas de higiene personal y ambiental deficientes, entre otras. A través de los patrones de salud funcional de Gordón se estructuraron los diagnósticos de enfermería; siendo el más relevante: conocimientos deficientes para mejorar la percepción del control de la salud y prevención de la enfermedad, relacionado con la deficiente información que reciben de sus padres y maestros. Se proponen cambios en programas de educación para la salud, favoreciendo el fomento, la prevención, control, tratamiento y referencia a servicios de salud.

Palabras clave: Diagnóstico de Enfermería. Preescolar. Salud.

\begin{abstract}
The conditions of life of the human being have changed, with it the health of etáreos groups. The human being exposes to practices of agreed health to its cultural patterns and the influence of the social networks. This study identified physical health of prestudents in suburban community of Toluca, Mexico. They were entrevisted to prestudents, counting on the authorization and is present at of the parents. The identified morbidity was in acute respiratory infections, gastrointestinales infectious diseases and malnutrición with predisponentes factors of deficient practices of personal and environmental hygiene, among others. Through the patterns of functional health of Gordón the diagnoses of nursing were structured; it was the main: deficient knowledge to improve the perception of the control of the health and prevention of the disease, related to the deficient information that receive from their parents and teachers. Changes in programs of education for the health set out, favoring the promotion, the prevention, control, treatment and reference to services of health.
\end{abstract}

\section{Resumo}

As condições de vida do ser humano têm sofrido transformações na sociedade e na saúde dos grupos etários. 0 indivíduo expõe as práticas de saúde em concordância com seus padrões culturais e a influência das redes sociais. Este estudo identificou a saúde física de pré-escolares em uma comunidade do município de Toluca, México. Foram entrevistados pré-escolares contando com autorização e presença dos pais. As doenças identificadas foram as infecções respiratórias agudas, infecciosas e gastrointestinais e subnutrição, com fatores predisponentes de práticas de higiene pessoal e ambiental deficientes, entre outras. Os padrões da saúde funcional de Gordón foram utilizados, e foram estruturados os diagnósticos da enfermagem, sendo os mais relevantes: os conhecimentos deficientes para a melhora da percepção do controle da saúde e prevenção da doença, relacionados com a deficiente informação que recebem de seus pais e professores. Propõem-se mudanças nos programas da instrução para a saúde, favorecendo a prevenção, o controle, o tratamento e a referência aos serviços da saúde. 


\section{INTRODUCCIÓN}

En cada etapa de desarrollo, el ser humano exige dirigir propias acciones, exponiéndose en forma intensa a prácticas específicas de salud en función de sus patrones culturales y la influencia percibida de las redes sociales, entendiendo que son aquéllas personas o grupos con las cuales mantiene contacto e influyen en su manera de pensar y actuar'. Este estudio se fundamento teóricamente en los patrones funcionales de Gordón ${ }^{2}$ y se identificaron los diagnósticos de enfermería ${ }^{3,4}$. Un patrón funcional se define como una expresión de integración psicosocial entre el cliente y el entorno y están influidos por factores biológicos, del desarrollo, culturales, sociales y espirituales, un patrón disfuncional puede aparecer con la enfermedad aunque también puede conducir a ella².

Las redes de apoyo social, son determinantes en el crecimiento, desarrollo y maduración de conocimientos, habilidades, actitudes y valores que las personas relacionan de manera directa con su salud. Los principales desafíos a la prolongación y calidad de la existencia del ser humano en las primeras etapas de la vida (grupos de riesgo), juegan un papel importante en determinados factores psicosociales como el estilo de vida, la cultura sanitaria, el estrés, el rol activo ante el cuidado y mantenimiento de su salud y la satisfacción con los sistemas de relaciones interpersonales en que esté inmerso el individuo. Todo ello es particularmente válido para la población convencionalmente conocida como infantil en este caso, los niños en edad preescolar ${ }^{5}$.

La información que el núcleo familiar brinda al preescolar y que a su vez se ve influenciada por otras redes sociales llamadas secundarias, no se percibe de manera adecuada y congruente a su contexto social e ideológico puesto que está delimitada e influenciada por diversos factores que incluyen el ambiente físico, social y cultural ${ }^{6}$.

Desafortunadamente tanto los mismos individuos como los adultos que los rodean desconocen el proceso de su desarrollo, sus características y manifestaciones, como la existencia de procesos de cambios socioculturales rápidos que afectan la familia, la sociedad, los valores, haciendo más complejas éstas etapas, lo que en ocasiones motiva a la adaptación de conductas equivocadas que pueden llevar a la enfermedad ${ }^{7}$.

Las últimas proyecciones de la Organización Panamericana de la Salud, de 2000 a 2006 muestran una Tasa de Mortalidad en menores de 5 años en la República Mexicana de $34.4 \%$, en el caso de las Infecciones Respiratorias Agudas, la proporción anual de defunciones registradas fue de $9 \%$ y se presentó como primera causa de muerte, así mismo la proporción anual de defunciones registradas por Enfermedades Diarreicas Agudas fue de $6 \%$, y se presentó como segunda causa de muerte 8 . Por otra parte, las Infecciones Respiratorias Agudas generan $29.2 \%$ de la consulta externa en los servicios de salud y $40 \%$ de las hospitalizaciones pediátricas. La ocurrencia de estos padecimientos en la comunidad, es similar durante todo el año; sin embargo, se identifica un incremento durante los meses más fríos ${ }^{8}$.

Los avances alcanzados en la prevención y control de las Infecciones Respiratorias Agudas, han sido notables, caso contrario de las enfermedades diarreicas y deficiencias nutricionales en donde cada día se encuentra mayor incidencia de malnutrición por deficiente ingesta calórico proteica, por escasos recursos económicos y por la falta de información y formación en cuanto a una dieta equilibrada en las comunidades semirurales y rurales. Para el Estado de México la tasa de mortalidad por cada 100,000 habitantes de ambos sexos en edad preescolar es de 6.5 para enfermedades infecciosas intestinales (CIE04), de 4.4 para mal nutrición (CIE06), y de 4.1 para infecciones respiratorias agudas (CIE07) ${ }^{9}$.

En el Estado de México, específicamente en el área comunitaria se ha visto la necesidad orientar a los preescolares, sobre prácticas positivas de salud que favorezcan su crecimiento y desarrollo; debido a que comienzan a experimentar situaciones propias de su etapa, las cuales repercuten directa e indirectamente sobre su estado de salud. Abordar la etapa preescolar desde la perspectiva de la salud permite un campo de acción más amplio y de mayor beneficio para estos individuos, ya que la principal tarea del profesional de enfermería es la promoción y prevención, motivando al cambio de conductas, actitudes y estilos de vida poco saludables, por otras que conduzcan al desarrollo de todo el potencial físico y psicosocial que hay en ellos ${ }^{10}$.

Los estudios acerca del estado de salud de la población y las intervenciones correspondientes del profesional de enfermería, deben dirigirse prioritariamente a los grupos más vulnerables. En la comunidad de San Pablo Autopan, en Toluca México los niños en edad preescolar, los de nivel primaria y los adolescentes presentan la mayor incidencia de morbilidad y prácticas poco saludables, considerándolas como el desconocimiento de acciones y actitudes en pro del autocuidado ${ }^{11}$. Con base en lo anterior el profesional de enfermería se ve en la necesidad de modificar su esquema de atención y considerar como prioritario, estudiar las condiciones de vida, hábitos y prácticas de mantenimiento de la salud en los diferentes estratos sociales, considerando la influencia de factores como el tipo de trabajo, ambiente, aspectos socioculturales, nivel educativo y económico de todo aquel que requiere de sus servicios.

La función de la enfermera en relación a la comunidad está encaminada a dirigir sus intervenciones al individuo, familia y colectividad, con un enfoque de prevención y promoción de la salud. Dentro de dichas intervenciones se incluye, la asesoría, educación, investigación y la provisión de servicios terapéuticos requeridos por parte de la población, así como la identificación e intervención en problemas de salud que estén dentro de sus posibilidades de acción de acuerdo a los medios con que se cuenta en el área comunitaria. De tal forma que a través de la elaboración de diagnósticos de salud tanto comunitarios como familiares e individuales la enfermera obtiene datos específicos que le orientan sobre como debe planear y ejecutar diferentes acciones encaminadas a la mejora continua del nivel y calidad de vida de la colectividad, así mismo cuente con la posibilidad de evaluar el impacto de sus intervenciones, revalorar, y modificar su plan de acción para dar seguimiento a los individuos y sus familias ${ }^{12}$

En la disciplina de enfermería el enfoque en la prestación de servicios a la población, se encuentra en estos momentos en un proceso evolutivo debido a los cambios sociales, culturales 
y políticos, los cuales señalan que actualmente se debe aplicar la salud comunitaria para fomento del autocuidado y prevención de la enfermedad ${ }^{12}$.

El propósito de esta investigación fue identificar el estado de salud física de la población preescolar a través de la aplicación de entrevistas y valoraciones físicas basadas en patrones de salud funcional de Gordón.

\section{METODOLOGÍA}

La investigación pertenece a la línea de salud comunitaria de la Facultad de Enfermería y Obstetricia de la Universidad Autónoma del Estado de México, particularmente al Programa Estado de Salud de la población ${ }^{13}$, en ella se identificó el estado de salud física de preescolares, en una comunidad suburbana de Toluca, México a través de la aplicación de entrevistas y valoraciones físicas basadas en Gordón ${ }^{2}$. Es un estudio cuanticualitativo, transversal y analítico. El universo fue un jardín de niños y la población en estudio los preescolares. Se entrevistaron a 73 preescolares, los criterios de inclusión fueron niños de 4 a 6 años que sus padres firmaron la hoja de consentimiento informado y estuvieron presentes durante la entrevista y valoración. Esto, permitió cubrir el aspecto bioético de la investigación por ser los sujetos de estudio menores de edad. El método para la recolección de datos se realizó a través de la aplicación de entrevistas y valoración física con base en los patrones de salud funcional de Gordón ${ }^{2}$. que incluyeron además datos generales de los padres. Para el análisis de los datos se generaron archivos por patrón funcional, se procedió al análisis de la información, identificando alguna alteración y se estructuraron los diagnósticos de enfermería ${ }^{3,4}$, proponiendo programas prioritarios de intervención.

\section{RESULTADOS Y DISCUCIÓN}

La infancia es un periodo biológico evolutivo del ser humano de extraordinaria importancia, que condiciona el resto de su vida, sobre todo porque en este momento el individuo sufre mayores cambios en cuanto a su crecimiento y desarrollo.

Al momento de la entrevista la mayoría de los preescolares negó la presencia de algún padecimiento; sin embargo se en la valoración que presentaban en ese momento síntomas de gripe y algunos de ellos refirieron dolor de garganta. Cubillos ${ }^{7}$, describe que desafortunadamente tanto los mismos individuos en etapa preescolar como los adultos que los rodean, desconocen el proceso de su desarrollo, sus características y manifestaciones, así como la existencia de procesos de cambios socioculturales rápidos que afectan la familia, la sociedad, los valores, haciendo más complejas estas etapas, lo que en ocasiones motiva la adaptación de conductas y actividades equivocadas que pueden llevar a la enfermedad.

En relación a la escolaridad de los padres se encontró que prácticamente la mitad de ellos cuentan con estudios concluidos de secundaria y los empleos a que tienen acceso son de albañil y obrero los cuales no son bien remunerados. Con respecto a las madres, un tercio de ellas cuenta con estudios de primaria y casi la totalidad se dedica al hogar. En el contexto social semirural y aún en vías de desarrollo de la comunidad en estudio, el núcleo familiar adopta un rol donde el padre es solamente proveedor de los recursos económicos y se deja el resto de las responsabilidades sobre el manejo y educación de los hijos a la madre que además de desempeñar el papel de padre- madre, tiene que fungir en varios roles, lo que no le permite llevar una vigilancia y control sobre la salud de sus hijos y las necesidades que puedan requerir de apoyo especial, por parte de alguno de los progenitores. Croft ${ }^{12}$ describe a la familia como red primaria y menciona que los padres son la fuerza educadora y el rol modelo para sus hijos, aunque las enfermeras y otros profesionales de la salud pueden ser indispensables para tratar y fomentar un rol preventivo en el individuo desde las primeras etapas de vida.

En la descripción de los patrones de la vida cotidiana se encontró que:

En el Patrón percepción - manejo de salud, en cuanto a la higiene personal del preescolar, la mayoría ellos se bañan cada tercer día y sólo una minoría muy marcada lo hace diariamente; en cuanto al cambio de ropa, este es diario en toda la población, así mismo casi el total de los preescolares acostumbra el aseo bucal dos veces al día y realiza el lavado de manos antes de comer y después de ir al baño. La mayoría de las madres refiere que utiliza un mismo espacio para dormir y cocinar debido a la carencia de recursos económicos necesarios para mantener independiente cada área. Croft ${ }^{12}$ menciona que no obstante la realidad en cuanto a información y formación que reciben los niños en edad preescolar, no es procesada congruentemente en la cotidianeidad de las familias puesto que está delimitada e influenciada por diversos factores como el tipo de casa habitación, el nivel socioeconómico, el grado de estudio de los padres, la distancia en cuanto a la ubicación de los servicios básicos de salud, los medios de transporte y la misma disponibilidad de tiempo con que cuenta cada uno de los integrantes del núcleo familiar, para dedicarse a la adquisición de conocimiento y habilidades que les permitan mejorar el autocuidado propio y transmitir los conocimientos adecuados a sus hijos.

La adopción de conductas saludables en el aspecto nutricional favorece al crecimiento y desarrollo del niño. Sin embargo, en la investigación se encontró que el preescolar adopta ciertos hábitos alimenticios que en ocasiones pueden alterar su estado de bienestar como ingerir alimentos no nutritivos entre comidas, evitar el consumo de verduras y legumbres, lo cual se ve reflejado en el aumento y disminución de peso en una tercera parte de la población. Más de un tercio de la población requiere de un seguimiento directo para control de su peso y talla. Turn ${ }^{14}$, señala que la nutrición adecuada es un factor vital para la salud, se incrementa la eficiencia, la satisfacción, el placer de la vida y el vigor de la futura generación. Las comidas deben ser variadas y al menos cuatro al día, sin descuidar las que contienen hierro, reforzar la incorporación de ensaladas con aporte vitamínico variado, preferir fruta fresca como postre, evitar las golosinas, alimentos fuera del horario, elegir el agua o jugos naturales sin azúcar como líquidos extras, limitando jugos artificiales y bebidas gaseosas.

Con respecto a la exploración física, se encontró que la mayor parte de los preescolares se encuentra en una talla 
promedio de 115.0 metros y un peso de 15 a 17 kilogramos. Para los niños de 4 a 6 años el peso adecuado debe ser entre los 18 kilogramos más menos 200 gramos y la talla se debe encontrar entre 101.3 a 113.2 metros. Según estándares para la población Mexicana ${ }^{15}$, los sujetos de estudio se encuentran fuera de los estándares normales al identificar bajo peso, lo que repercute a mediano o largo plazo en consecuencias graves para el menor. De ahí la importancia de implementar programas específicos que atiendan este tipo de problemas en el aspecto nutricio de la población preescolar, en donde la enfermera promueva los hábitos adecuados de alimentación no sólo con los niños sino también con los padres y profesores que son quienes forman al preescolar.

En cuanto a la salud bucal, casi la mitad de la población presenta algún grado de caries. Es importante promover entre los padres de familia, el que induzcan al preescolar a mantener una higiene bucal constante y realizarla de manera correcta, pues con esto se evitaría complicaciones a futuro ya que no sólo afectan la estética del niño sino también su autoestima, además de que en algunos casos se pueden requerir tratamientos específicos de ortodoncia que representan un gran desembolso a la economía de la familia y Tomey ${ }^{16}$, menciona que la familia es considerada como la estructura básica de la sociedad y constituye el primer grupo de apoyo social, cuya función es la de orientar a sus miembros sobre los valores y estilos de vida a través de las experiencias sociales y patrones de interacción. Sin embargo, los padres tienen una errónea concepción del papel como formadores que desempeñan frente al niño en crecimiento y esto los lleva a pensar que la educación que los hijos reciben en las escuelas, será lo que les permita adquirir cierta inmunidad ante cualquier tipo de enfermedad y eventualidad que se presente en el transcurso de su desarrollo hacia la madurez; así mismo las autoridades educativas en el caso específico de la población en estudio abordan de manera general el tema de la salud y las enfermedades más frecuentes en esta etapa de la vida, pues no están capacitados para hacerlo de manera correcta además de que lo consideran de incumbencia familiar y médica, limitándose a una interacción superficial lo que trae como consecuencia la no identificación temprana de patologías que pueden afectar a la totalidad de la comunidad preescolar.

Los diagnósticos de enfermería encontrados en los preescolares y que se consideran de prioridad son los siguientes: - Déficit de autocuidado en la alimentación, relacionado con bajos recursos económicos y falta de información de fuentes nutricionales de bajo costo; evidenciado por el número de niños con bajo peso y estatura en relación a su edad. (Código 00102)

- Déficit de autocuidado en el baño e higiene personal, relacionado con las costumbres culturalmente aprendidas; evidenciado por el aspecto observado en los niños al momento de interrogarlos y explorarlos. (Código 00108)
- Conocimientos deficientes para mejorar la percepción del control de la salud y prevención de la enfermedad, relacionado con la deficiente información que reciben de sus padres y maestros, evidenciado por la presencia de infecciones respiratorias agudas y enfermedades diarreicas. (Código 00126)

- Deterioro de la dentición, relacionado con prácticas de higiene bucal deficientes y falta de atención dental; evidenciado por el número de niños con caries y piezas dentarias perdidas y dañadas por la misma causa. (Código 00048)

Los resultados demuestran que si bien es cierto existen diversas patologías que pueden ser prevenibles en edades tempranas con una buena orientación sobre prácticas saludables por parte de las principales redes de apoyo social (padres, maestros y personal de salud), se requiere de intervención específica del personal de enfermería que favorezca el autocuidado a través de la aplicación de programas de educación para la salud, en los cuales se transmita la información adecuada y acorde con los conocimientos que sobre la enfermedad tienen los escolares, padres de familia y maestros, y se planeen y ejecuten acciones que impacten en elevar el estado de bienestar de la población vulnerable.

Debido a la trascendencia de los resultados obtenidos, se considera prioritario realizar intervenciones de enfermería en la Prevención de Infecciones Respiratorias Agudas, enfermedades infecciosas gastrointestinales, y mal nutrición en preescolares, con lo cual se pretende diseñar proyectos de investigación que den respuesta a las necesidades de salud identificadas. Acorde a esta problemática en los sujetos de estudio y con sustento en la Ley General de Salud 2001-2006 17 se proponen programas prioritarios de promoción de conductas saludables en las que se incluye la prevención de enfermedades propias de un rezago epidemiológico que no se ha logrado abatir, y que por las características propias de la región de estudio siguen considerándose entre las primeras causas de morbilidad y mortalidad en población vulnerable. Las enfermedades son las Infecciones respiratorias agudas, Infecciones gastrointestinales y Malnutrición.

\section{CONCLUSIONES}

Las primeras causas de morbilidad identificadas en los sujetos de estudio son infecciones respiratorias agudas, enfermedades infecciosas gastrointestinales y malnutrición. En el presente estudio se identificó la presencia de factores que inciden en la adquisición de conductas no saludables como deficiente higiene personal y ambiental y deficiente información nutricional, que contribuyen para este cuadro epidemiológico.

Los preescolares saben más de lo que practican, ya que a pesar de conocer medidas de autocuidado y los riesgos de enfermedad sus prácticas difieren cuando se compara su estado de salud.

Es necesaria la intervención de enfermería en los programas de educación para la salud para reforzar las conductas saludables de los preescolares. 


\section{Referencias}

1. Dabas E. Las prácticas de las intervenciones en redes sociales. México(DF) Paidos;1993. p.189.

2. Gordon M. Diagnóstico enfermero, proceso y aplicación, $3^{\mathrm{a}}$ ed. Madrid (ES): Mc-Graw Hill Interamericana; 1999. p. 457

3. NANDA. International, Diagnósticos enfermeros: definiciones y clasificación 2003-2004. Madrid(ES): Elsevier; 2004.

4. NANDA International. Diagnósticos enfermeros: definiciones y clasificación 2005-2006. Madrid (ES): Mc-Graw Hill Interamericana ; 2006.

5. Alvaro J. Psicología social aplicada. Madrid(ES): Mc-Graw Hill Interamericana; 1996. p. 536.

6. Kroeger A. Atención primaria de salud: principios y métodos. México(MX): OPS; 1992.

7. Cubillos L. Salud del adolescente. Facultad de Enfermería de la Universidad Nacional de Colombia. Avances en Enfermería 1993; 11 (1): 29-35.

8. Organización Panamericana de la Salud-OPS. Perfil del sistema de servicios de salud en México. Boletín Informativo; 2004.

9. Instituto de Salud del Estado (MX). Departamento de Estadística. Sistema Estadístico y Epidemiológico de
Defunciones (CIE-10). Principales causas de mortalidad en preescolares. México(MX); 2005

10. Cruz B. Estudio de comunidad y diagnóstico de salud de San Mateo Otzacatipan, Municipio de Toluca. México (MX): Facultad de Enfermería y Obstetricia/ UAEM; 2003.

11. Hall J. Enfermería en salud comunitaria: un enfoque de sistemas. México(MX): OPS; 1990.

12. Croft C, Asmussen L. A developmental approach to sexuality education: implications for medical practice. J Adolesc Health 1993; 14:109-14.

13. Cruz B, Cárdenas B, Arizmendi Arizmendi, et al. Formalización de énfasis, líneas y programas de investigación de la Facultad de Enfermería y Obstetricia/UAEM. Toluca(MX); 1996. Documento de trabajo de la F E y 0 .

14. Turn CE. Higiene del individuo y de la comunidad. Limusa (MX); 1989.

15. Instituto Nacional de Estadística Geografía e InformáticaINEGI (MX). Estadísticas y índice de desnutrición en el Estado de México; 2005.

16. Tomey AM, Alligood MR. Modelos y teorías en enfermería. $4^{a}$ ed. Madrid (ES): Mosby; 1999.

17. Ley General de Salud (MX). Disponible en: http:// www.eean.ufrj.br/docs/normas2004.pdf 